\title{
At the Helm of the Burning Ship
}

\author{
Claude Heiland-Allen \\ unaffiliated \\ London, UK \\ claude@mathr.co.uk
}

\begin{abstract}
The Burning Ship fractal is a non-analytic variation of the Mandelbrot set, formed by taking absolute values in the recurrence. Iterating its Jacobian can identify the period of attracting orbits; Newton's root-finding method locates their mini-ships. Size estimates tell how deep to zoom to find the mini-ship or its embedded quasi-Julia set. Pre-periodic Misiurewicz points with repelling dynamics are located by Newton's method. Stretched regions are automatically unskewed by the Jacobian, which is also good for colouring images using distance estimation. Perturbation techniques cheapen deep zooming. The mathematics can be generalised to other fractal formulas. Some artistic zooming techniques and domain colouring methods are also described.
\end{abstract}

Burning Ship. Dynamical systems. Fractal art. Numerical algorithms. Perturbation theory.

\section{IMAGING THE BURNING SHIP}

\subsection{ESCAPE-TIME FRACTALS}

Fractals are mathematical objects exhibiting detail at all scales. Escape-time fractals are plotted by iterating recurrence relations parameterised by pixel coordinates from a seed value until the values exceed an escape radius $R$ or until an arbitrary limit on iteration count $N$ is reached (this is to ensure termination, as some pixels may not escape at all). The colour of each pixel is determined by how quickly the iterates took to escape.

Define the integer pixel coordinates by $i, j$, with the centre of the image at $i_{c}, j_{c}$, then the viewing transformation for a region near $a_{c}, b_{c}$ can be factored using polar decomposition of its $2 \times 2$ matrix (Uhlig 1981) into a uniform scale factor $s$, a rotation $r$, and a non-uniform stretch $\kappa$ where $\operatorname{det} \kappa=1:$

$$
\left(\begin{array}{l}
a \\
b
\end{array}\right)=\left(\begin{array}{l}
a_{c} \\
b_{c}
\end{array}\right)+\operatorname{sr\kappa }\left(\left(\begin{array}{l}
i \\
j
\end{array}\right)-\left(\begin{array}{l}
i_{c} \\
j_{c}
\end{array}\right)\right)
$$

\subsection{The Burning Ship}

The most famous escape-time fractal is the Mandelbrot set, with the complex-valued recurrence relation $z_{n+1}=z_{n}^{2}+c$, where $c$ is based on the pixel coordinates and $z_{0}=0$.

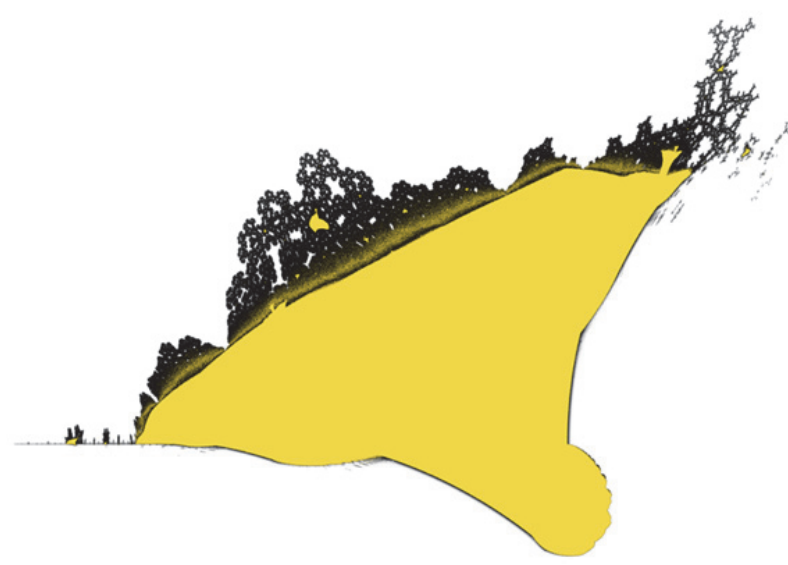

Figure 1: The Burning Ship fractal. $a \in[-2.02,1.18], b \in$ $[-1.8,0.6]$

The Burning Ship fractal defined by Michelitsch \& Rössler (1992) modifies this formula with the addition of absolute values, which makes the formula non-analytic (it must be considered as a pair of real-valued recurrences):

$x_{n+1}=x_{n}^{2}-y_{n}^{2}+a \quad y_{n+1}=2\left|x_{n}\right|\left|y_{n}\right|+b \quad x_{0}=y_{0}=0$ Unlike the Mandelbrot set, the Burning Ship is typically plotted with the $b$ axis increasing in the downwards direction: this makes the fractal shape resemble a ship on fire. 


\subsection{Renormalised iteration count}

Plotting the escape iteration count alone leads to images with banded level sets. However, the size of the final iterate (the first that escaped beyond $R$ ) can be used for smooth colouring. The renormalised fractional iteration count Vepstas (1997) derived for the Mandelbrot set is also valid for the Burning Ship as its asymptotic behaviour towards infinity is the same:

$$
\mu=n+1-\log _{2}\left(\log \sqrt{x_{n}^{2}+y_{n}^{2}}\right)
$$

\subsection{Screen-space distance estimates}

Approaching the boundary of an escape-time fractal, the iteration bands get closer and closer together. This happens in such a way that an estimate of the distance to the boundary can be calculated. The $\mu$ values of neighbouring pixels combined together with Roberts' cross operator gives a number that is smaller than 1 near the boundary and greater than 1 far from the boundary, which is useful information for colouring images with the structure of the fractal:

$$
d_{i, j}=\frac{1}{\sqrt{\left|\mu_{i, j}-\mu_{i+1, j+1}\right|^{2}+\left|\mu_{i, j+1}-\mu_{i+1, j}\right|^{2}}}
$$

\subsection{Analytic distance estimates}

The Mandelbrot set has a well-known analytic exterior distance estimate based on derivatives. "gerrit" (2017) adapted it to the Burning Ship by replacing the complex derivatives with a vectorbased norm of the Jacobian matrix (with respect to $a, b)$, which I combined with the viewing transform:

$$
\begin{gathered}
\left(\begin{array}{ll}
u & v
\end{array}\right)=\left(\begin{array}{ll}
x_{n} & y_{n}
\end{array}\right) J_{n} s r \kappa \\
d_{i, j}=d_{0} \frac{\left(x_{n}^{2}+y_{n}^{2}\right) \log \sqrt{x_{n}^{2}+y_{n}^{2}}}{\sqrt{u^{2}+v^{2}}}
\end{gathered}
$$

Setting $d_{0}=s$ gives an estimate of the distance to the fractal boundary, setting $d_{0}=1$ gives a value scaled to screen-space.

The Jacobian is a matrix of derivatives:

$$
J_{n}=\left(\begin{array}{ll}
\frac{\partial x_{n}}{\partial a} & \frac{\partial x_{n}}{\partial b} \\
\frac{\partial y_{n}}{\partial a} & \frac{\partial y_{n}}{\partial b}
\end{array}\right)
$$

The derivatives can be calculated using recurrence relations, defining $\frac{\partial|z|}{\partial z}=\operatorname{sgn} z$. I set $\operatorname{sgn} 0=1$ to avoid some issues with singular $J_{n}$ on the negative $a$ axis (elsewhere $\operatorname{sgn} z=\frac{z}{|z|}$ ).

$$
\begin{gathered}
\frac{\partial x_{n+1}}{\partial a}=2 x_{n} \frac{\partial x_{n}}{\partial a}-2 y_{n} \frac{\partial y_{n}}{\partial a}+1 \\
\frac{\partial x_{n+1}}{\partial b}=2 x_{n} \frac{\partial x_{n}}{\partial b}-2 y_{n} \frac{\partial y_{n}}{\partial b} \\
\frac{\partial y_{n+1}}{\partial a}=2\left|x_{n}\right| \operatorname{sgn} y_{n} \frac{\partial y_{n}}{\partial a}+2 \operatorname{sgn} x_{n} \frac{\partial x_{n}}{\partial a}\left|y_{n}\right| \\
\frac{\partial y_{n+1}}{\partial b}=2\left|x_{n}\right| \operatorname{sgn} y_{n} \frac{\partial y_{n}}{\partial b}+2 \operatorname{sgn} x_{n} \frac{\partial x_{n}}{\partial b}\left|y_{n}\right|+1 \\
0=\frac{\partial x_{0}}{\partial a}=\frac{\partial x_{0}}{\partial b}=\frac{\partial y_{0}}{\partial a}=\frac{\partial y_{0}}{\partial b}
\end{gathered}
$$

Whether to use screen-space or analytic distance estimates is an aesthetic choice: different locations may expose different strengths and weaknesses. Computationally, analytic distance estimation may seem more costly due to the extra Jacobian calculations, however it might need a lower supersampling ratio for a high quality image.

\section{FEATURES OF INTEREST}

\subsection{Mini-ships}

The Burning Ship fractal contains mini-ships, which resemble smaller copies of the whole. Borrowing terminology from Mandelbrot set literature, the nucleus of the mini-ship is an attracting periodic point $(a, b)$, such that $x_{p}=y_{p}=0$ for some $p>0$. The smallest such $p$ is called the period of the miniship.

\subsubsection{Finding periods}

An algorithm to find the lowest period of a miniMandelbrot set in a region by iterating the corners of a polygon is described by Munafo (2008) but this cannot be applied to the Burning Ship without extensive modifications because of the non-analytic folding by the absolute values in the Burning Ship formula.

An alternative method based on a technique I learnt from "knighty" (2017) is to iterate the centre of the region under along with its Jacobian (with respect to $(a, b))$ and detect the first period $p$ when:

$$
\left\|\left(J_{p} s r \kappa\right)^{-1}\left(x_{p} \quad y_{p}\right)\right\|<1
$$

This is similar to iterating an implicitly-defined ellipse instead of a polygon.

\subsubsection{Finding mini-ships}

Newton's root-finding method in one complex variable is a standard technique for finding periodic points in the Mandelbrot set. I applied the less wellknown multidimensional version to the Burning Ship. 
Given the period $p$ of a mini-ship, and an approximation to its nucleus $\left(a_{0}, b_{0}\right)$, one can use the method to solve for $(a, b)$ such that $x_{p}=y_{p}=0$. Multidimensional Newton's method uses the Jacobian to refine a solution using an implicit recurrence relation:

$$
J_{p}\left(\begin{array}{l}
a_{m+1}-a_{m} \\
b_{m+1}-b_{m}
\end{array}\right)=-\left(\begin{array}{l}
x_{p} \\
y_{p}
\end{array}\right)
$$

Here $J_{p}$ and $\left(x_{p}, y_{p}\right)$ are evaluated using $\left(a_{m}, b_{m}\right)$ at each step of Newton's method.

\subsubsection{The size of mini-ships}

I adapted the Mandelbrot size estimate presented by Hunt \& Ott (1997) to the Burning Ship with minor adjustments, using Jacobian matrices (this time with respect to $(x, y)$ instead of $(a, b))$ to replace the complex derivatives. The partial derivative recurrences are:

$$
\begin{gathered}
\frac{\partial x_{n+1}}{\partial x_{1}}=2 x_{n} \frac{\partial x_{n}}{\partial x_{1}}-2 y_{n} \frac{\partial y_{n}}{\partial x_{1}} \\
\frac{\partial x_{n+1}}{\partial y_{1}}=2 x_{n} \frac{\partial x_{n}}{\partial y_{1}}-2 y_{n} \frac{\partial y_{n}}{\partial y_{1}} \\
\frac{\partial y_{n+1}}{\partial x_{1}}=2\left|x_{n}\right| \operatorname{sgn} y_{n} \frac{\partial y_{n}}{\partial x_{1}}+2 \operatorname{sgn} x_{n} \frac{\partial x_{n}}{\partial x_{1}}\left|y_{n}\right| \\
\frac{\partial y_{n+1}}{\partial y_{1}}=2\left|x_{n}\right| \operatorname{sgn} y_{n} \frac{\partial y_{n}}{\partial y_{1}}+2 \operatorname{sgn} x_{n} \frac{\partial x_{n}}{\partial y_{1}}\left|y_{n}\right| \\
1=\frac{\partial x_{1}}{\partial x_{1}}=\frac{\partial y_{1}}{\partial y_{1}} \\
0=\frac{\partial x_{1}}{\partial y_{1}}=\frac{\partial y_{1}}{\partial x_{1}}
\end{gathered}
$$

and the resulting size estimate $s_{M}$ evaluated at a nucleus of period $p$ is:

$$
\begin{gathered}
L_{n}=\left(\begin{array}{ll}
\frac{\partial x_{n}}{\partial x_{1}} & \frac{\partial x_{n}}{\partial y_{1}} \\
\frac{\partial y_{n}}{\partial x_{1}} & \frac{\partial y_{n}}{\partial y_{1}}
\end{array}\right) \quad \beta=\sum_{n=1}^{p-1} L_{n}^{-1} \quad S=\beta L_{p}^{2} \\
s_{M}=\frac{1}{\sqrt{|\operatorname{det} S|}}
\end{gathered}
$$

\subsubsection{Examples}

The largest ship has period 1 and nucleus at $(0,0)$. Its size is 0.143 . The largest mini-ship on the negative $a$ axis has period 3 and nucleus at $(-1.7549,0)$. Its size is 0.00865 . The largest miniship on the top right of the main ship has period 3 and nucleus at $(0.87744,-1.5198)$. Its size is 0.00941 .

\subsection{Misiurewicz points}

In the Mandelbrot set, pre-periodic Misiurewicz points with repelling dynamics are found at the tips of filaments and the centres of spirals, and also other places. In the Burning Ship, they occur in similar locations.

\subsubsection{Finding Misiurewicz points}

Like strictly periodic points, Misiurewicz points can be found using Newton's method for root finding when given the pre-period $q$ and period $p$, this time solving:

with the iteration:

$$
x_{q+p}=x_{q} \quad y_{q+p}=y_{q}
$$

$$
\left(J_{q+p}-J_{q}\right)\left(\begin{array}{l}
a_{m+1}-a_{m} \\
b_{m+1}-b_{m}
\end{array}\right)=-\left(\left(\begin{array}{l}
x_{q+p} \\
y_{q+p}
\end{array}\right)-\left(\begin{array}{l}
x_{q} \\
y_{q}
\end{array}\right)\right)
$$

\subsubsection{The multiplier of Misiurewicz points}

In the Mandelbrot set, the multiplier of a Misiurewicz point is the complex derivative of its periodic cycle. This provides a scale factor for the asymptotic self-similarity of the Mandelbrot set near the Misiurewicz point.

In the Burning Ship, the derivative is replaced by a Jacobian matrix (with respect to $(x, y)$ ), and the multiplier is then:

$$
m=\left(\begin{array}{ll}
\frac{\partial x_{q+p}}{\partial x_{q}} & \frac{\partial x_{q+p}}{\partial y_{q}} \\
\frac{\partial y_{q+p}}{\partial x_{q}} & \frac{\partial y_{q+p}}{\partial y_{q}}
\end{array}\right)
$$

A similarity scale factor is then $\sqrt{\mid \operatorname{detm|}}$; rotation and non-uniform stretch can be extracted using polar decomposition.

\subsubsection{Examples}

$(-2,0)$ has pre-period 2, period 1, and multiplier $\left(\begin{array}{ll}4 & 0 \\ 0 & 4\end{array}\right)$. Zooming in by a factor of 4 gives a similar image.

$(0,-1)$ has pre-period 2 , period 2 , and multiplier $\left(\begin{array}{cc}4 & 4 \\ -4 & 4\end{array}\right)$. Zooming in by a factor of $4 \sqrt{2}$ with a rotation of 45 degrees gives a similar image, though this is not visible with most colouring algorithms as the point is inside the main bulk of the ship shape.

$(0.82443,-1.6657)$ has pre-period 3 , period 1 , and multiplier $\left(\begin{array}{cc}2.5412 & -2.1617 \\ 2.1617 & 2.5412\end{array}\right)$. Zooming in by a factor of 3.3362 with a rotation of 40.387 degrees gives a similar image, though in this case the images are noticeably stretched. Setting $\kappa$ to the optimal skew matrix for a nearby escaping point (as defined in the following section) enhances the similarity. 


\subsection{Unskewing stretched areas}

Images of the Mandelbrot set are typically rendered with a 1:1 pixel aspect ratio. However, some regions of the Burning Ship look rather stretched out when visualised this way, so a compensating reverse stretch matrix $\kappa$ can be applied to the parameter values before iteration.

Using the matrix $\beta$ as defined in the mini-ship size estimate, the optimal skewing matrix for a region around a mini-ship is $\kappa=\beta^{\Lambda_{1}}$, where the hat indicates normalisation such that $|\operatorname{det} \kappa|=1$.

For regions where it is too hard to find a mini-ship, because it is already too stretched to navigate coherently, a skew matrix can be calculated from the Jacobian (with respect to $a, b$ ) of a nearby point that escapes: $\kappa=J_{n}^{\Lambda_{1}}$.

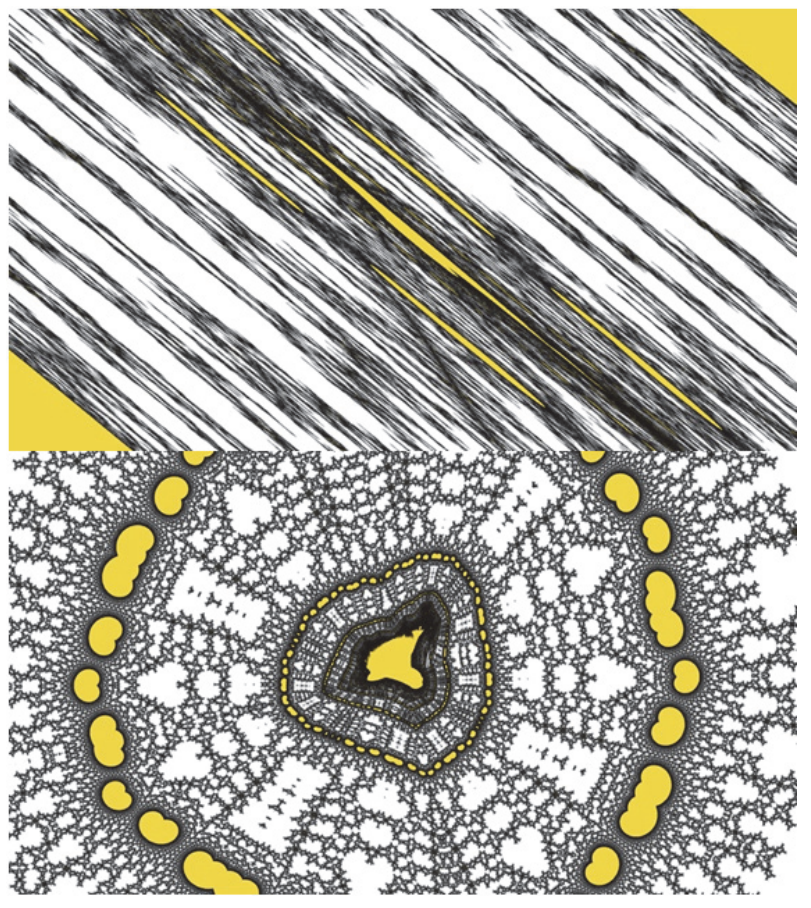

Figure 2: Comparison of identity transformation and optimal skew transformation.
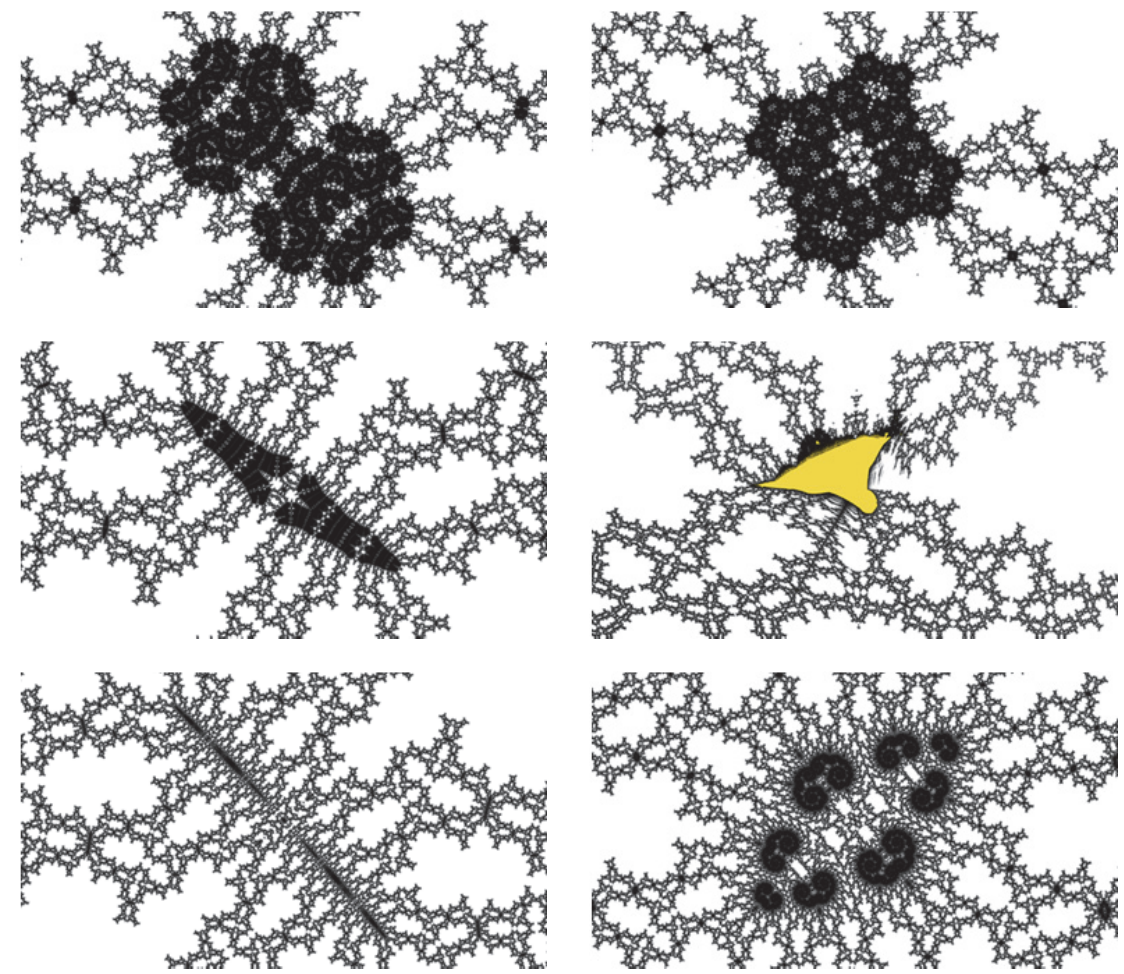

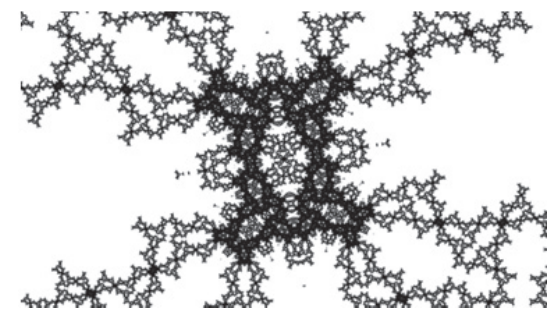

Figure 3: Embedded Julia sets in the filaments surrounding a mini-ship. 


\subsection{Embedded Julia sets}

Embedded Julia sets in the Mandelbrot set are an interesting phenomenon, wherein zooming around half way between one mini and another, deeper, mini reveals a structure in the filaments that resembles a Julia set from the inner location relative to the outer mini (Jung 2018). Julia sets are related to the Mandelbrot set by setting $z_{0}$ based on the coordinates of each pixel, this time setting $c$ to a constant for the whole image.

A similar thing happens in the Burning Ship. The embedded quasi-Julia set between an outer miniship of size $s_{O}$ and an inner mini-ship of size $s_{I}$ has a size $s_{J}$ approximately $s_{J}=\sqrt{s_{O} s_{I}}$. If only the inner size is known, a rough estimate for the size of the embedded Julia set can be found by $s_{J}=s_{I}^{\frac{3}{4}}$.

\section{DEEP ZOOM RENDERING}

\subsection{Perturbation}

Martin's SuperFractalThing popularised perturbation techniques for the Mandelbrot set (2013). With deep zooming one needs higher precision numbers, calculations with which take more time. The perturbation technique is to iterate a single high precision reference orbit, along with low precision deltas for each pixel. Using exact algebra with the unevaluated sums, most of the "large" values fall out, which allows the "small" deltas to be updated without catastrophic loss of significance.

"laser blaster" (2014) constructed a case analysis for evaluating $|X+x|-|X|$ without losing precision when $|X| \gg|x|$ (naïvely, $X+x \approx X$ when only a few significant digits are available, so the evaluation would always give 0 ):

$$
\operatorname{diffabs}(X, x)=\left\{\begin{array}{cc}
x & X \geq 0 \wedge X+x \geq 0 \\
-(2 X+x) & X \geq 0 \wedge X+x<0 \\
2 X+x & X<0 \wedge X+x>0 \\
-x & X<0 \wedge X+x \leq 0
\end{array}\right.
$$

Then the perturbed iterations $(a, b, x, y)$ for the Burning Ship with reference $(A, B, X, Y)$ are:

$$
\begin{gathered}
x_{n+1}=2 X_{n} x_{n}+x_{n}^{2}-2 Y_{n} y_{n}-y_{n}^{2}+a \\
y_{n+1}=2 \operatorname{diffabs}\left(X_{n} Y_{n}, X_{n} y_{n}+x_{n} Y_{n}+x_{n} y_{n}\right)+b
\end{gathered}
$$

If the reference escapes early, one needs to choose another reference and recalculate the remaining pixels.

Perturbation does not need to be applied to the Jacobian for distance estimation as there are no issues of cancellation here.

\subsection{Perturbation glitches}

It can be that the dynamics of some pixels are sufficiently different to the dynamics of the reference, such that there is precision loss and visible glitches. "Pauldelbrot" (2014) constructed a criterion to detect this case in the Mandelbrot set, the same principle applies to the Burning Ship: if $\left(X_{n}+x_{n}\right)^{2}+\left(Y_{n}+y_{n}\right)^{2}<10^{-3}\left(X_{n}^{2}+Y_{n}^{2}\right)$ then a problem may occur. In this case a good candidate new reference may be in a pixel which minimises $\left(X_{n}+x_{n}\right)^{2}+\left(Y_{n}+y_{n}\right)^{2}$, at the iteration number at which the glitch is detected. This is based on the observation that glitches tend to surround miniships with the glitch occurring at an iteration which is a multiple of the period.

\subsection{Series approximation}

Martin's SuperFractalThing, as well as using perturbation, also uses series approximation techniques to speed up Mandelbrot set graphical rendering. However, when applied to the Burning Ship, special care must be taken if the image of the region to be rendered ever crosses an axis, because then the non-analytic absolute value part of the Burning Ship formula will fold it into multiple parts on the next iteration. No single series can account for more than one of these parts, so the region must be split along the fold lines, with a separate series needed for each. More research is needed to see if the complexity of implementation is worth it.

\section{GENERALISATIONS}

The degree $d \in N$ Burning Ship generalisation modifies the iteration recurrence to:

$$
x_{n+1}+i y_{n+1}=\left(\left|x_{n}\right|+i\left|y_{n}\right|\right)^{d}+(a+i b)
$$

Here $i=\sqrt{-1}$ is the imaginary unit. The normalised iteration count becomes:

$$
\mu=n+1-\log _{d}\left(\log \sqrt{x_{n}^{2}+y_{n}^{2}}\right)
$$

where $d$ is the highest non-linear power in the iteration formula, corresponding to behaviour near infinity. The derivatives in the equations for the Jacobian recurrences can be calculated for higher powers by straightforward algebraic manipulation. The size estimate for mini-sets needs to be changed to:

$$
s_{M}=\frac{1}{\sqrt{|\operatorname{det} \beta|} \sqrt{\left|\operatorname{det} L_{p}\right|^{\frac{d}{(d-1)}}}}
$$

where $d$ is the lowest non-linear power in the formula, corresponding to behaviour near 0 . The rough embedded Julia set size estimate becomes

$$
s_{J}=s_{I}^{\frac{(d+1)(d-1)}{d^{2}}}
$$


The more accurate embedded Julia set size estimate is a weighted geometric mean:

$$
s_{J}=\sqrt[d]{s_{O} S_{I}^{d-1}}
$$

The techniques in this paper are not limited to the Burning Ship. They also work for other fractal formulas (for example, "Celtic", "Buffalo" or "Mandelbar") with straightforward algebraic manipulation which I have automated by a computer algebra system.

\section{ARTISTIC TECHNIQUES}

\subsection{Sculptural zooming}

The path taken when zooming into fractals such as the Mandelbrot set or the Burning Ship determines the structures visible in the image at different zoom depths. By making choices about where to zoom, a fractal artist can sculpt desired shapes from the mathematical structure.

\subsubsection{Shape stacking}

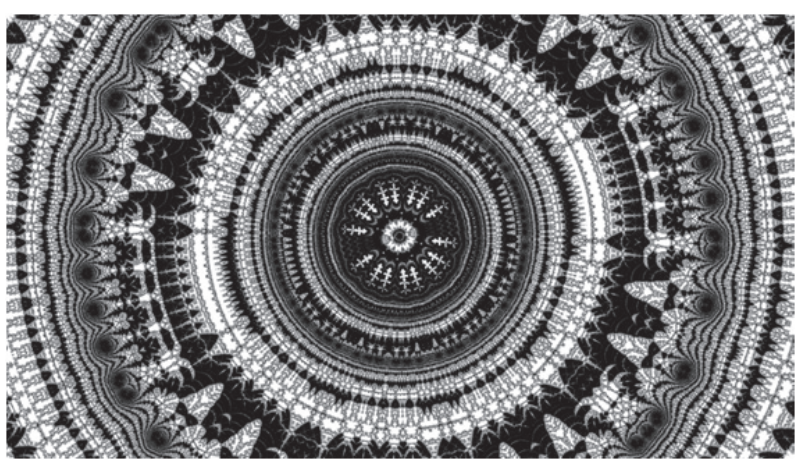

Figure 4: Shape stacking.

Shape stacking can be achieved by zooming close to a mini-ship in the outer decorations of a miniship in the outer decorations of a mini-ship (and so on). The shapes from each embedded Julia set passed through on the zoom path, stack up in concentric period-doubling rings.

\subsubsection{Layer stacking}

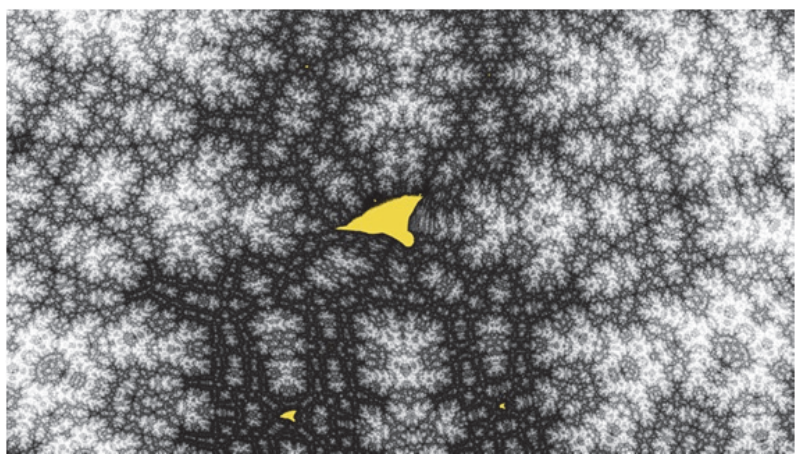

Figure 5: Layer stacking.
Layer stacking can be achieved by zooming close to a mini-ship in the inner decorations of a mini-ship in the inner decorations of a mini-ship (and so on). Mapping the logarithm of the distance estimate into a linear colour palette can give aesthetically pleasing effects, particularly for these dense deep zooms (Runmo 2017).

\subsubsection{Julia morphing}

Julia morphing can be achieved by zooming close to a feature of an embedded Julia set deeper to its "echo", where the feature is wrapped twice around the centre. "Going off centre" at this point close to a feature of this new structure, repeatedly alternating with zooming in a regular procedure, can lead to diverse shapes.

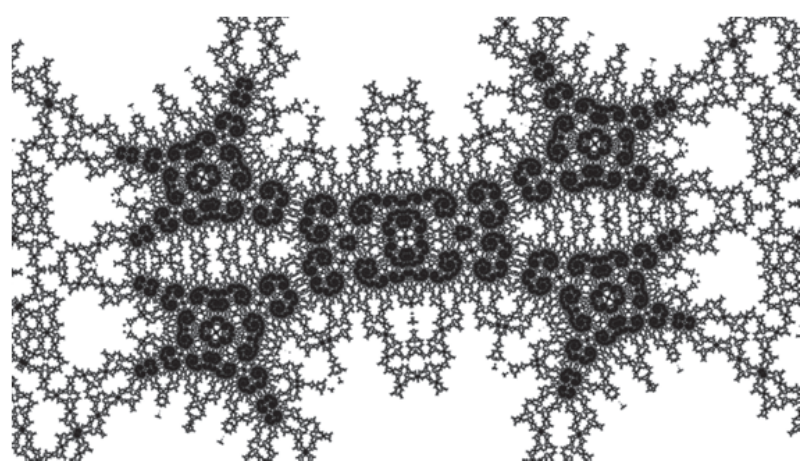

Figure 6: Julia morphing.

\subsection{Structural colouring}

\subsubsection{Atom domains}

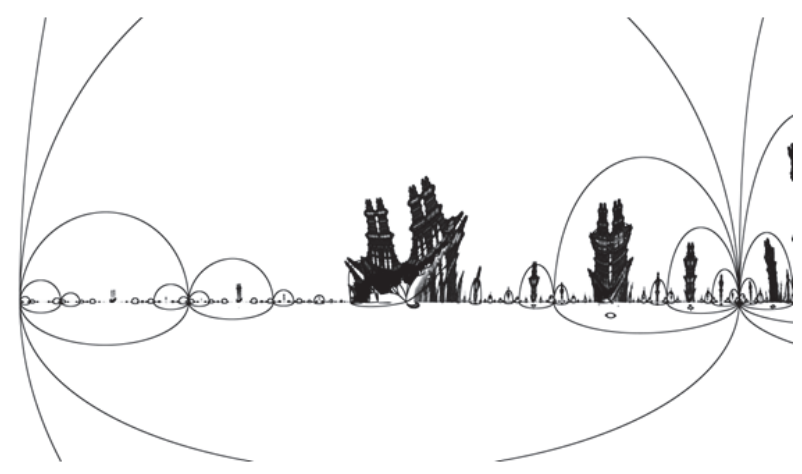

Figure 7: Atom domain colouring variation.

Atom domain colouring as defined by Peitgen \& Richter (1986, pp61-62) for the Mandelbrot set is to colour points based on the iteration count $m$ at which $x_{m}^{2}+y_{m}^{2}$ is minimised. For points that escape to infinity this is well-defined, and even in the interior it seems stable, revealing previously unseen structures. Atom domains surround miniships of the corresponding period, but are usually significantly larger, which can aid in navigation. 


\subsubsection{Misiurewicz domains}

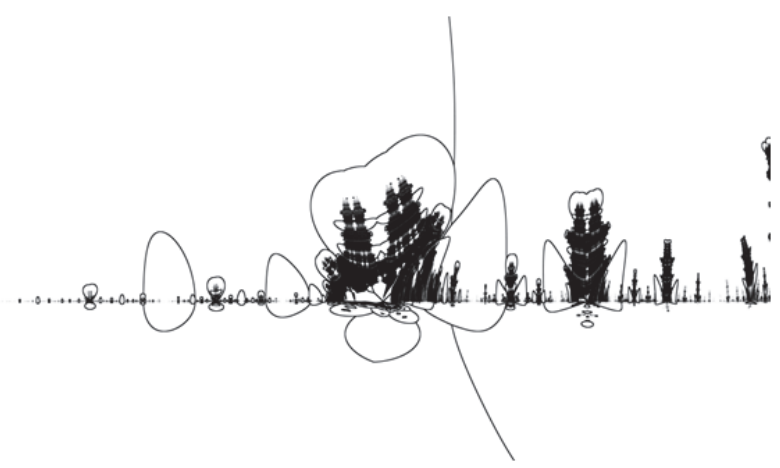

Figure 8: Misiurewicz domain colouring variation.

I extended the atom domain idea to Misiurewicz domain colourings. For a period $p$ this is to colour points based on the iteration count $q$ at which $\left(x_{q+p}-x_{q}\right)^{2}+\left(y_{q+p}-y_{q}\right)^{2}$ is minimised, welldefined for exterior points that escape to infinity. The domains surround Misiurewicz points of preperiod $q$ and period $p$. The best periods to visualise depend on the location within the fractal.

\section{Phantom domains}

Unfortunately some domains seem to be phantoms, with neither mini-ship nor Misiurewicz boundary point at their centre. This may be down to the lack of analyticity, as this problem doesn't seem to occur in the Mandelbrot set.

\section{CONCLUSION}

The Burning Ship and other fractal formulas are not as well-behaved as the Mandelbrot set. The latter's dynamics of one complex variable have been studied in great depth, with much useful machinery like external rays. Non-analytic formulas do still admit some artistically useful algorithms: one can let the computer do the uninteresting work of finding precise coordinates, zoom depths and skew matrices, leaving more free time for higher-level artistic choices

My experimental work-in-progress implementation et with a fractal formula compiler and runtime (running on Linux systems only), is available at https://mathr.co.uk/et/.

A fixed collection of formulas is compiled using et into my fork of Kalle's Fraktaler 2 (for Windows systems, including WINE), which is available at https://mathr.co.uk/kf/kf.html.

\section{ACKNOWLEDGEMENTS}

I am indebted to the community at Fractal Forums (both dot-org and formerly dot-com) for insightful discussions about ongoing research topics in the field of escape time fractals. Thanks also to the anonymous reviewers for their constructive comments.

\section{REFERENCES}

"gerrit" (2017) Re: burning ship distance estimation. https://fractalforums.org/f/18/t/647/msg3207\#msg3 207 (retrieved 14 February 2019).

Hunt, B. R., and Ott, E. (1997) Structure in the Parameter Dependence of Order and Chaos for the Quadratic Map. J. Phys. A 30, 7067-7076.

Jung, W. (2018) Renormalization and embedded Julia sets in the Mandelbrot set. Preprint of December 2018.

http://mndynamics.com/papers/embed.pdf (retrieved 14 February 2019)

"knighty" (2017) A little note about Ball arithmetic. https://fractalforums.org/f/28/t/481/msg2800\#msg2 $\underline{800}$ (retrieved 14 February 2019)

"laser blaster" (2014) Perturbation Formula for Burning Ship. http://www.fractalforums.com/index.php?topic $=191$ 65.msg74089\#msg74089 (retrieved 14 February 2019).

Martin, K. I. (2013) SuperFractalThing Maths. http://www.superfractalthing.co.nf/sft maths.pdf (retrieved 14 February 2019).

Michelitsch, M. and Rössler, O. E. The "Burning Ship" and its Quasi-Julia sets. Comput. \& Graphics vol.16, no4, 1992, pp.435-438.

Munafo, R. (2008) Finding the Period of a muAtom.

https://www.mrob.com/pub/muency/period.html (retrieved 14 February 2019).

"Pauldelbrot" (2014) Perturbation Theory Glitches Improvement.

http://www. fractalforums.com/index.php?topic $=189$ 08.msg73027\#msg73027

(retrieved 14 February 2019).

Peitgen, H. G. and Richter, P. H. (1986) The Beauty of Fractals: Images of Complex Dynamical Systems. Springer-Verlag, Berlin Heidelberg.

Runmo, K. (2017) Re: compiling Kalles Fraktaler with mingw. http://www. fractalforums.com/index.php?topic $=254$ 58.msg103177\#msg103177 (retrieved 14 February 2019).

Uhlig, F. (1981) Explicit polar decomposition and a near-characteristic polynomial: The $2 \times 2$ case. Linear Algebra and its Applications Volume 38, June 1981, pp.239-249.

Vepstas, L. (1997) Renormalizing the Mandelbrot Escape. https://linas.org/artgallery/escape/escape.html (retrieved 14 February 2019). 